\title{
Einleitung und Ausgangslage
}

\author{
„Wenn man nicht weiß, \\ welchen Hafen man ansteuern soll, \\ ist kein Wind der richtige.“ (Seneca 1991, S. 19)
}

\subsection{Problemstellung und Relevanz der internationalen Zielmarktanalyse und Vertriebsaufbau bzw. Vertriebsentwicklung in internationalen Märkten}

Das Zitat von Seneca fasst eine Seite der Problemstellung der Dissertation auf anschauliche Weise zusammen. Viele kleinere und mittelständische Unternehmen (im Nachfolgenden bezeichnet als KMUs) aber auch Großunternehmen (im Nachfolgenden bezeichnet als GUs) fragen sich, welche neuen Märkte sie mit welchen Produkten ,ansteuern“ sollen. Die andere Seite der Problemstellung der Dissertation betrifft die Ankunft im Hafen, wenn man das Zitat von Seneca erweitern würde. Welche Markteintrittspunkte gibt es, d. h. woher bekomme ich Informationen zu diesem neuen Markt? Welche Markteintrittsstrategie passt zu meinem Unternehmen und wie baue ich erste Vertriebswege auf, um meine Produkte vom Hafen in die Städte bzw. zu den Kunden zu bekommen? Diese und weitere Fragen werden mit der vorliegenden Dissertation beantwortet.

Dass die aufgezeigte Situation eine reale Problemstellung für Unternehmen bedeutet, verdeutlicht Abb. 1.1. Der Praxis fehlt es an geeigneten Modellen zur Internationalisierung der Geschäfte. Ein interviewter Geschäftsmanager z. B. fragt sich, wie er nach der Marktauswahl verfahren soll, um erste Geschäfte im ausgewählten Zielmarkt zu tätigen. Es gebe keine geeigneten Modelle, die erklären, wie Unternehmen bei der Erschließung ausländischer Märkte vorgehen. (siehe Zitat 1 
in Abb. 1.1). Weiterhin werden die Internationalisierungsaufgaben auf verschiedene Funktionen im Unternehmen verteilt, was zu Schnittstellenproblemen führt (siehe Zitat 2 in Abb. 1.1). Eine mögliche Lösung des Schnittstellenproblems kann z. B. darin bestehen, dass die Aufgaben nicht nach Funktionen aufgeteilt werden, sondern nach einem Prozess ${ }^{1}$. In der Forschung wird auch auf das Problem des Schnittstellenmanagements hingewiesen. Panagopoulos et al. sehen eine Lücke in Bezug auf das Schnittstellenmanagement in Unternehmen zwischen den Abteilungen Vertrieb und anderen funktionellen Bereichen ${ }^{2}$. Wagner \& SzymuraTyl argumentieren, dass die Schnittstellen zwischen den Abteilungen Vertrieb, Marketing und Neuproduktentwicklung erforscht werden müssen ${ }^{3}$.

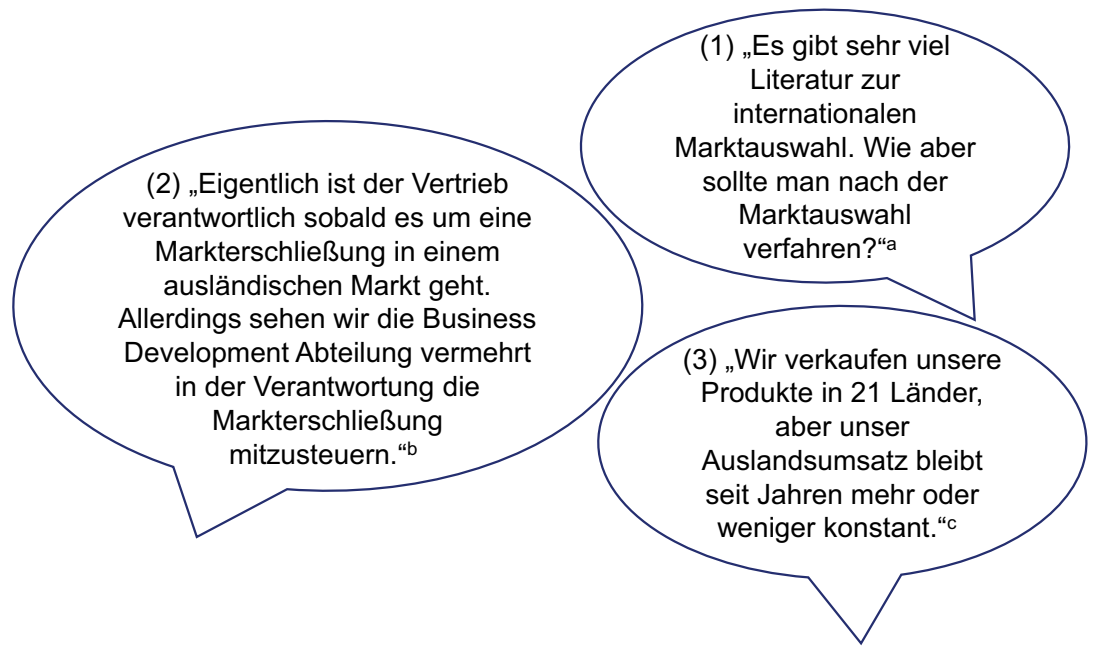

Abb. 1.1 Problemstellungen in der Praxis. ${ }^{\mathrm{a}}$ Interview 3 2016; ${ }^{\mathrm{b}}$ Interview 16 2016; ${ }^{\mathrm{c}}$ Interview 7 2016. (Quelle: Eigene Darstellung)

Andere Unternehmen wiederum erschließen mehrere Ländermärkte, da z. B. Anfragen für Produktlieferungen aus den Märkten kommen, aber der Umsatz in dem Ländermarkt wird nicht weiter ausgebaut (siehe Zitat 3 in Abb. 1.1).

\footnotetext{
${ }^{1}$ Vgl. Krüger 2011, S. 213 ff.

${ }^{2}$ Vgl. Panagopoulos et al. 2011, S. 227.

${ }^{3}$ Vgl. Wagner \& Szymura-Tyl 2016, S. 3619 f.
} 
Meistens bleibt es bei einem einmaligen Umsatz oder es entsteht eine Geschäftsverbindung zu einem ausländischen Unternehmen das dauerhaft beliefert wird. ${ }^{4}$

Insgesamt zeigen die geführten Experteninterviews, dass sowohl einige KMUs als auch einige GUs nicht systematisch genug bei der Internationalisierung ihrer Geschäfte vorgehen ${ }^{5}$. Durch diese unstrukturierte Vorgehensweise werden Umsatzpotenziale in ausländischen Märkten vernachlässigt bzw. gar nicht erst abgeschöpft. Das ISA-Modell setzt an der aufgezeigten Problematik an. Es zeigt in einem sieben-stufigen Internationalisierungsprozess-Modell wie Unternehmen ihr individuelles Internationalisierungsmuster ganzheitlich planen und implementieren können.

\subsection{Zielsetzung der Arbeit}

Aufgrund der beschriebenen Problemstellung lassen sich die in Abb. 1.2 dargestellten Forschungsfragen und Forschungsziele ableiten.

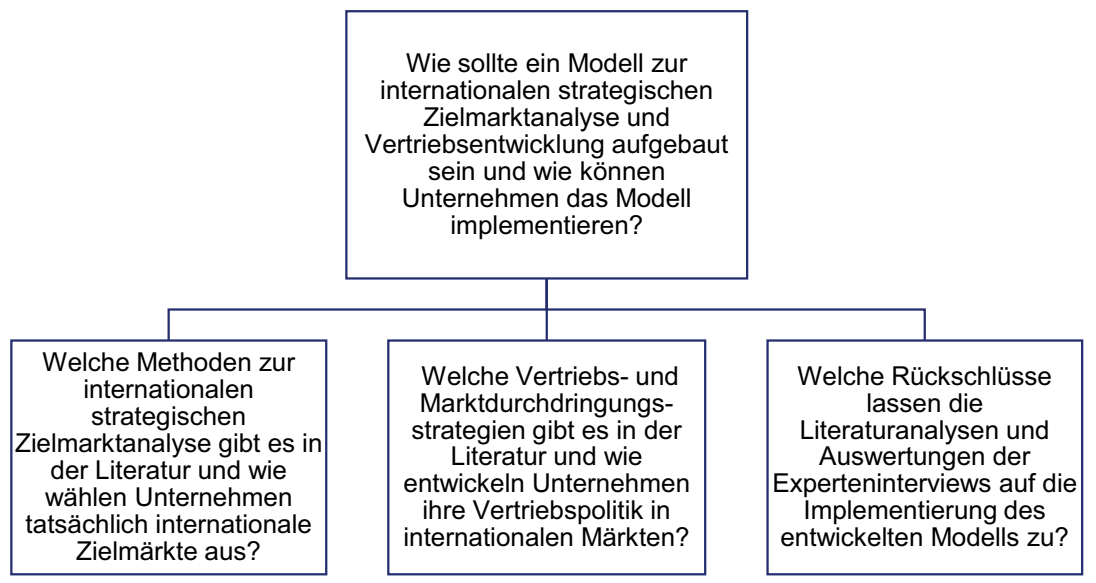

Abb. 1.2 Forschungsfragen der Dissertation. (Quelle: Eigene Darstellung in Anlehnung an Minto 1987, S. 9)

\footnotetext{
${ }^{4}$ Vgl. z. B. Interview 242016 oder Interview 102015.

${ }^{5}$ Siehe z. B. Tab. 5.1 und 5.2 in Kapitel 5.
} 
Das übergeordnete Ziel der vorliegenden Dissertation ist, ein Modell zur internationalen strategischen Zielmarktanalyse und zur internationalen Vertriebsentwicklung aufzustellen sowie Managern erste Ansätze zu liefern, wie sie dieses Modell anwenden können (siehe Ebene 1 in Abb. 1.2). Die Forschungsunterfragen (siehe Ebene 2 in Abb. 1.2) sollen zur Beantwortung der Forschungsoberfrage beitragen und deren Komplexität herunterbrechen. Forschungsunterfrage eins und Forschungsunterfrage zwei sind Recherchefragen, die durch Sekundär- und Primärdaten beantwortet werden können. Ziel ist es den Forschungsstand zu den Themen Marktauswahl und Vertriebs- sowie Marktdurchdringungsstrategien aufzuarbeiten und eigene Forschungsergebnisse zu präsentieren. Forschungsunterfrage drei ist eine Hypothesenfrage, die durch die Verknüpfung der Erkenntnisse aus den Forschungsunterfragen eins und zwei beantwortet werden kann. Alle drei Forschungsunterfragen führen zur Beantwortung der Forschungsoberfrage. Mit dem entwickelten Modell wird das Ziel verfolgt, eine Forschungslücke zu füllen (siehe Kapitel 2) und sowohl für die Wissenschaft als auch für die Praxis einen substanziellen Beitrag zu leisten.

\subsection{Beschreibung der Forschungsmethodik}

Die Untersuchungsgegenstände der Dissertation sind zum einen Internationalisierungsmuster, d. h. das konkrete Vorgehen eines einzelnen Unternehmens bei der Internationalisierung seiner Geschäfte und zum anderen der Internationalisierungsprozess, d. h. die abstrakten Ebenen, die alle Unternehmen bei einer Internationalisierung der Geschäfte durchlaufen.

Die Gestaltung der Datenerhebung und Datenanalyse erfolgte anhand der qualitativen Grounded Theory-Methode ${ }^{6}$ und der qualitativen InhaltsanalyseMethode $^{7}$ unter Zuhilfenahme der Software MAXQDA. Mit Hilfe der Grounded Theory wurden die Bausteine des ISA-Modells durch eine induktive Vorgehensweise entwickelt ${ }^{8}$. Das nachfolgende Zitat erklärt die Vorgehensweise bei Grounded Theory-Studien:

\footnotetext{
"A grounded theory is one that is inductively derived from the study of the phenomenon it represents. That is, it is discovered, developed, and provisionally verified through systematic data collection and data analysis of data pertaining to that phenomenon. Therefore, data collection, analysis, and theory stand in reciprocal
}

\footnotetext{
${ }^{6}$ Vgl. Glaser \& Strauss 1967 und Vgl. Strauss \& Corbin 1990.

${ }^{7}$ Vgl. Mayring 2015.

${ }^{8}$ Vgl. Strauss \& Corbin 1990, S. 23.
} 
relationship with each other. One does not begin with a theory, then prove it. Rather, one begins with an area of study and what is relevant to that area is allowed to emerge." 9

Wie aus dem Zitat hervorgeht, gibt es eine reziproke Beziehung zwischen der Datenerhebung und der Datenanalyse. Aus diesem Grund wurde über drei Jahre hinweg in vier verschiedenen Ländern nach und nach Daten erhoben (siehe Abb. 1.3).
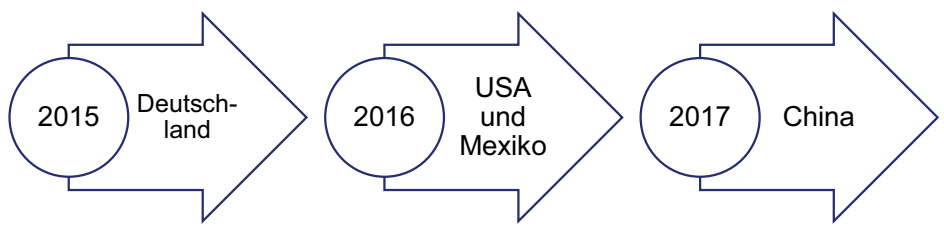

Abb. 1.3 Zeitliche Durchführung der Interview-Datenerhebung. (Quelle: Eigene Darstellung)

Die ersten teilstrukturierten Interviews fanden im Rahmen eines Drittmittelprojektes für das Land Baden-Württemberg im Jahr 2015 statt. Anschließend wurden weitere teilstrukturierte Interviews im Rahmen von Forschungsaufenthalten in den USA, Mexiko und China durchgeführt. Die Forschungsaufenthalte wurden durch Stipendien finanziert: Rudi-Häussler Förderpreis (USA), Strategisches Netzwerk Bioökonomie (BECY) (Mexiko) und Thematisches Netzwerk Innovation, Entrepreneurship und Finanzierung (INEF) (China).

Die Gestaltung des Interviewleitfadens wurde mehrmals während der Betreuung von Seminararbeiten am Lehrstuhl für Internationales Management an der Universität Hohenheim durch die Autorin vorab getestet. Der Interviewleitfaden wurde auf Deutsch und auf Englisch verfasst. Je nach Interviewpartner wurde das Interview entweder auf Deutsch oder auf Englisch geführt. Der entstandene Interviewleitfaden enthält drei Kategorien: A. Allgemeine Fragen zur Person und zum Unternehmen, B. Fragen zum Internationalisierungsprozess und C. Rückblick und abschließende Fragen (siehe separater und vertraulicher Anhang; siehe auch Hinweis im Quellenverzeichnis). Der im separaten und vertraulichen Anhang enthaltene Fragebogen enthält die Ausgangsfragen. Je nach Interviewpartner und fortschreitendes Wissen der Autorin wurden Follow-up Fragen gestellt. Insgesamt

\footnotetext{
${ }^{9}$ Strauss \& Corbin 1990, S. 23.
} 
wurde bei der Frageformulierung die grundlegenden Anforderungen an Frageformulierungen nach $K u \beta$ et al. beachtet ${ }^{10}$. Außerdem wurde darauf geachtet möglichst offene Fragen zu formulieren, um der interviewten Person genug Zeit zum Erzählen zu ermöglichen ${ }^{11}$. Eine Beispielfrage lautet: Wie haben Sie einen für Sie besonders erfolgreichen Auslandsmarkt erschlossen ${ }^{12}$ ?

Insgesamt wurden 79 Interviews von jeweils 1-2 Stunden durchgeführt. 47 Interviews wurden durch die Autorin selbst geführt und transkribiert. Weitere 37 Interviews wurden von Studierenden nach Einarbeitung durch die Autorin durchgeführt und transkribiert. Mit manchen Unternehmen wurden mehrere Interviews abgehalten. Für die Datenanalyse wurden letztendlich 54 Unternehmen ausgewählt (siehe separater und vertraulicher Anhang; siehe separater und vertraulicher Anhang; siehe auch Hinweis im Quellenverzeichnis). Die restlichen 25 Interviews waren entweder Interviews mit Institutionen oder Zweitgespräche mit Interviewpartnern. Alle Zweitgespräche von einem Unternehmen wurden bei der Auswertung berücksichtigt und beim ersten Interview mit eingearbeitet.

Nachdem das ISA-Modell durch kontinuierliche Verfeinerung entwickelt war, wurde mit Hilfe der Methode der qualitativen Inhaltsanalyse von Mayring jedes Interview nach Tätigkeiten gescannt und den zuvor aufgestellten Schritten des ISA-Modells zugeordnet ${ }^{13}$. $\mathrm{Zu}$ Beginn der Auswertung mussten relativ viele Codes aufgestellt werden. Je mehr Interviews analysiert waren, umso weniger wurden neue Codes nötig. Im Anschluss an die erste Auswertung wurden die Codes auf logische Zusammenhänge überprüft und ggfs. weiter zusammengefasst ${ }^{14}$. Das Ergebnis der Auswertung der qualitativen Inhaltsanalyse findet sich zusammengefasst in Abb. 5.1, dem Schnell-Check zur Einschätzung des eigenen Internationalisierungsmusters im ISA-Modell. In den einzelnen Kapiteln zum ISA-Modell werden die Ergebnisse der Auswertung ebenfalls ausführlich beschrieben.

Neben der Datenerhebung von Primärdaten wurden auch Daten von Sekundärquellen herangezogen. Besonders nennenswert sind z. B. zwei Datenbanken, die mit einer Lizenz durch die Universität Hohenheim benutzt werden können. Die erste Datenbank nennt sich Orbis-Datenbank und ist vom Bureau von Dijk ${ }^{15}$. Die Auswahl der Umwelttechnikunternehmen erfolgte durch die NACE Rev. 2, einer

\footnotetext{
${ }^{10}$ Vgl. Kuß et al. 2014, S. 83 ff.

${ }^{11}$ Vgl. Andresen 2013.

${ }^{12}$ siehe separater und vertraulicher Anhang; siehe auch Hinweis im Quellenverzeichnis

${ }^{13}$ Vgl. Mayring 2015, S. 70.

${ }^{14}$ Vgl. Mayring 2015, S. $70 \mathrm{ff}$.

${ }^{15}$ Vgl. Bureau van Dijk 2015.
} 
statistischen Systematik der Wirtschaftszweige ${ }^{16}$, und der Datenbank Orbis ${ }^{17}$. Der Schwerpunkt auf die Umwelttechnikindustrie wurde durch ein Drittmittelprojekt mit dem Land Baden-Württemberg gesetzt. In Tab. 1.1 sind die Kerncodes für drei ausgewählte Segmente der Umwelttechnik abgebildet. Nachdem die NACE Rev. 2 Codes identifiziert waren, wurde mit Hilfe der Datenbank Orbis Umwelttechnikunternehmen in vier ausgewählten Ländern, Deutschland, USA, China und Mexiko identifiziert.

Tab. 1.1 Übersicht über die Segmente der Umwelttechnikindustrie sowie der verwendeten NACE Rev. 2 Codes

\begin{tabular}{l|c|l}
\hline $\begin{array}{l}\text { Segmente der } \\
\text { Umwelttechnikindustrie }\end{array}$ & $\begin{array}{l}\text { NACE } \\
\text { Rev. 2 } \\
\text { Codes }\end{array}$ & Beschreibung \\
\hline $\begin{array}{l}\text { Abfall- und } \\
\text { Kreislaufwirtschaft }\end{array}$ & 38 & $\begin{array}{l}\text { Sammlung, Behandlung und Beseitigung } \\
\text { von Abfällen; Rückgewinnung }\end{array}$ \\
\hline$($ Ab-) Wasserwirtschaft & 36 & Wasserversorgung \\
\cline { 2 - 3 } & 37 & Abwasserentsorgung \\
\hline Luftreinhaltung & 2825 & $\begin{array}{l}\text { Herstellung von kälte- und lufttechnischen } \\
\text { Erzeugnissen, nicht für den Haushalt }\end{array}$ \\
\cline { 2 - 3 } & 2829 & $\begin{array}{l}\text { Herstellung von sonstigen nicht } \\
\text { wirtschaftszweigspezifischen Maschinen }\end{array}$ \\
\cline { 2 - 3 } & 2899 & $\begin{array}{l}\text { Herstellung von Maschinen für sonstige } \\
\text { bestimmte Wirtschaftszweige }\end{array}$ \\
\cline { 2 - 3 } & 4669 & $\begin{array}{l}\text { Großhandel mit sonstigen Maschinen und } \\
\text { Ausrüstungen }\end{array}$ \\
\cline { 2 - 3 } & & andation \\
\hline
\end{tabular}

Quellen: Eigene Darstellung in Anlehnung an Statistisches Amt der Europäischen Union 2008, S. 93 ff.

Bei qualitativen Studien ist es wichtig eine möglichst diverse Stichprobe $\mathrm{zu}$ bekommen ${ }^{18}$. Deswegen wurden weitere Interviews mit Unternehmen aus möglichst vielen unterschiedlichen NACE Kern-Code Industrien und weiteren Heimatländern geführt (siehe Tab. 1.2). Weiterhin wurde darauf geachtet, dass unterschiedlich große Unternehmen in der Stichprobe vorhanden sind. Dabei

\footnotetext{
${ }^{16} \mathrm{Vgl}$. Statistisches Amt der Europäischen Union (im Nachfolgenden bezeichnet als Eurostat) 2008.

${ }^{17}$ Vgl. Bureau van Dijk 2015.

${ }^{18}$ Vgl. Schatzinger et al. 2015, S. 6 f.
} 
wurden die Unternehmen nach der KMU-Definition der Europäischen Union eingeteilt: Unternehmen mit einer Mitarbeiterzahl bis zu 249 Personen und einem Jahresumsatz bis zu 50 Mio. EUR wurden als KMU eingestuft. Unternehmen mit einer Mitarbeiterzahl von 250 Personen und einem Jahresumsatz von 51 Mio. EUR wurden als GUs eingestuft. ${ }^{19}$ Weitere Interviews wurden mit Institutionen, wie z. B. den Außenhandelskammern oder Wirtschaftsförderungsgesellschaften geführt.

Tab. 1.2 Übersicht über NACE Rev. 2 Codes in anderen Industrien

\begin{tabular}{|c|c|}
\hline $\begin{array}{l}\text { NACE } \\
\text { Rev. } 2 \\
\text { Codes }\end{array}$ & Beschreibung \\
\hline 1920 & Mineralölverarbeitung \\
\hline 2342 & Herstellung von Sanitärkeramik \\
\hline 2593 & Herstellung von Drahtwaren, Ketten und Federn \\
\hline 2712 & Herstellung von Elektrizitätsverteilungs- und -schalteinrichtungen \\
\hline 2751 & Herstellung von elektrischen Haushaltsgeräten \\
\hline 2811 & $\begin{array}{l}\text { Herstellung von Verbrennungsmotoren und Turbinen (ohne Motoren für } \\
\text { Luft- und Straßenfahrzeuge) }\end{array}$ \\
\hline 2813 & Herstellung von Pumpen und Kompressoren \\
\hline 2814 & Herstellung von Armaturen \\
\hline 2822 & Herstellung von Hebezeugen und Fördermitteln \\
\hline 2893 & $\begin{array}{l}\text { Herstellung von Maschinen für die Nahrungs- und Genussmittelerzeugung } \\
\text { und die Tabakverarbeitung }\end{array}$ \\
\hline 2910 & Herstellung von Kraftwagen und Kraftwagenmotoren \\
\hline 2932 & Herstellung von sonstigen Teilen und sonstigem Zubehör für Kraftwagen \\
\hline 3299 & Herstellung von sonstigen Erzeugnissen \\
\hline 4322 & Gas-, Wasser-, Heizungs- sowie Lüftungs- und Klimainstallation \\
\hline 4329 & Sonstige Bauinstallation \\
\hline 4520 & Instandhaltung und Reparatur von Kraftwagen \\
\hline 4637 & Großhandel mit Kaffee, Tee, Kakao und Gewürzen \\
\hline 4652 & Großhandel mit elektronischen Bauteilen und Telekommunikationsgeräten \\
\hline 5229 & Erbringung von sonstigen Dienstleistungen für den Verkehr \\
\hline
\end{tabular}

(Fortsetzung)

${ }^{19}$ Vgl. Institut für Mittelstandsforschung Bonn 2018. 
Tab. 1.2 (Fortsetzung)

\begin{tabular}{l|l}
\hline $\begin{array}{l}\text { NACE } \\
\text { Rev. 2 } \\
\text { Codes }\end{array}$ & Beschreibung \\
\hline 7010 & Verwaltung und Führung von Unternehmen und Betrieben \\
\hline 7111 & Architekturbüros \\
\hline 7739 & Vermietung von sonstigen Maschinen, Geräten und beweglichen Sachen \\
\hline 9609 & Erbringung von sonstigen Dienstleistungen \\
\hline
\end{tabular}

Quellen: Eigene Darstellung in Anlehnung an Statistisches Amt der Europäischen Union 2008, S. 93 ff.

Die zweite Datenbank ist die United Nations Comtrade Datenbank von den Vereinten Nationen ${ }^{20}$. In der Datenbank werden durch die Vereinten Nationen Export- und Importzahlen aller durch die Weltzollorganisation gekennzeichneten Produkte gesammelt und können anhand von Codes für Produkte oder bei mehreren Codes für Industrien abgerufen werden (siehe Fallstudie 6.1). Weitere Sekundärquellen wurden z. B. für die Literaturanalysen benötigt. Diese wurden ebenfalls über Fachdatenbanken, wie z. B. Business Source Premier identifiziert und über die Lizenz der Universität Hohenheim sowie der University of Texas at Austin heruntergeladen. Da die vorliegende Dissertation mehrere in der Regel unabhängig voneinander betrachtete Themen vereint, wurde bei der Literatursuche auf die Stichwort-Erfahrung von Prof. Gillespie von der University of Texas at Austin zurückgegriffen. Mit ihrer Hilfe wurden passende Stichworte zur Literatursuche ausgewählt. Weitere Sekundärquellen waren Unternehmensbroschüren, die die Interviewpartner zur Verfügung stellten. Für die Fallstudien wurde sowohl single-case Designs als auch multiple-case Designs nach Yin ausgewählt ${ }^{21}$.

\subsection{Aufbau der Arbeit}

Kapitel 2 bettet das ISA-Modell in die Business Development und Vertriebsforschung ein (Abschn. 2.1) und stellt die Entwicklung der Methodik des ISA-Modells vor (Abschn. 2.2). Es werden theoretische Grundlagen und Definitionen vorgestellt und das Thema der Arbeit zu anderen wissenschaftlichen Bereichen abgegrenzt und eingeordnet.

\footnotetext{
${ }^{20}$ Vgl. United Nations Comtrade Datenbank 2015.

${ }^{21}$ Vgl. Yin 2009, S. 46.
} 
In Kapitel 3 werden die Ergebnisse aus den Experteninterviews zu den ISAModell Bausteinen 1-3 dargestellt (Abschn. 3.1-3.3). Die Bausteine 1-3 des ISA-Modells gehören zur strategischen Analyse im Prozess des strategischen Managements.

Kapitel 4 erläutert die Ergebnisse aus den Experteninterviews in den ISAModell Bausteinen 4-7 (Abschn. 4.1-4.4). Baustein 4 repräsentiert dabei den Teil der Strategieformulierung und Strategieauswahl im Prozess des strategischen Managements. Die Bausteine 5-7 stehen für die Strategieimplementierung im Prozess des strategischen Managements.

In Kapitel 5 werden die Ergebnisse der Experteninterviews zusammengefasst. Es wird ein Schnell-Check zur Selbsteinschätzung des eigenen Internationalisierungsmusters für Manager vorgestellt (Abschn. 5.1) und im Anschluss auf die Implementierung der Methodik des ISA-Modells eingegangen (Abschn. 5.2). Hierbei wird die in Kapitel 2 vorgestellte Literatur mit den in Kapitel 3 und 4 vorgestellten Ergebnissen aus den Experteninterviews verknüpft und gemäß Forschungsunterfrage 3 Rückschlüsse für die Implementierung des ISA-Modells gezogen.

Kapitel 6 zeigt konkrete Vorgehensweisen für die Zielmarktanalyse für eine Industrie (Abschn. 6.1) und unterschiedliche Internationalisierungsmuster von Unternehmen (Abschn. 6.2). In Abschn. 6.1 wird eine eigenständig entwickelte Methode für eine Zielmarktanalyse in einer Querschnittsindustrie wie der Umwelttechnikindustrie vorgestellt. Dabei wird auf die ersten drei Bausteine des ISA-Modells eingegangen. Abschn. 6.2 stellt die Internationalisierungsmuster von zehn Unternehmen vor, dabei wird auf alle Bausteine des ISA-Modells eingegangen. Die Fallstudien in Abschn. 6.2 sind so ausgewählt, dass möglichst unterschiedliche Markteintrittsstrategien und deren Implementierung beschrieben werden können (siehe auch Abb. 6.9).

In Kapitel 7 werden die Ergebnisse der Dissertation kurz und prägnant zusammengefasst und mit der anfangs aufgestellten Zielsetzung verglichen (Abschn. 7.1). Anschließend werden Empfehlungen für die Weiterentwicklung des ISA-Modells gegeben und Hypothesen für zukünftige Follow-up Studien zum ISA-Modell dargestellt (Abschn. 7.2). Zu guter Letzt wird auf Limitierungen der Dissertation eingegangen und einen Ausblick auf zukünftige Forschungsvorhaben unabhängig vom ISA-Modell gegeben (Abschn. 7.3). 
Open Access Dieses Kapitel wird unter der Creative Commons Namensnennung 4.0 International Lizenz (http://creativecommons.org/licenses/by/4.0/deed.de) veröffentlicht, welche die Nutzung, Vervielfältigung, Bearbeitung, Verbreitung und Wiedergabe in jeglichem Medium und Format erlaubt, sofern Sie den/die ursprünglichen Autor(en) und die Quelle ordnungsgemäß nennen, einen Link zur Creative Commons Lizenz beifügen und angeben, ob Änderungen vorgenommen wurden.

Die in diesem Kapitel enthaltenen Bilder und sonstiges Drittmaterial unterliegen ebenfalls der genannten Creative Commons Lizenz, sofern sich aus der Abbildungslegende nichts anderes ergibt. Sofern das betreffende Material nicht unter der genannten Creative Commons Lizenz steht und die betreffende Handlung nicht nach gesetzlichen Vorschriften erlaubt ist, ist für die oben aufgeführten Weiterverwendungen des Materials die Einwilligung des jeweiligen Rechteinhabers einzuholen.

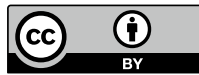

